\title{
Some Remarks on the Abstract Cauchy Problem
}

\author{
By \\ Nobuhiro SANEKATA*
}

\section{Introduction}

This paper is concerned with the abstract Cauchy problem

$A C P$

$$
\left\{\begin{aligned}
\frac{d}{d t} u(t) & =A u(t) \quad 0 \leqq t<T \\
u(0) & =x
\end{aligned}\right.
$$

for a linear operator $A$ (with domain $D(A)$ and range $R(A)$ ) in a Banach space $X$.

Let $T \in(0, \infty], x \in X$ and $u(t)=u(t ; x) ;[0, T) \rightarrow X$. Then $u(t)$ is called a solution to $(A C P ; A, T, x)$ if $u(t)$ is strongly continuously differentiable in $t \in[0, T), u(t) \in D(A)$ for $t \in[0, T)$ and $u(t)$ satisfies $A C P$. If there is no ambiguity, we use abbreviations $(A C P ; T, x), A C P$ instead of $(A C P ; A, T, x)$. If, in addition, $T=\infty$ and $u(t)$ satisfies, for a fixed $\omega \in R$,

$$
\|u(t)\|+\left\|u^{\prime}(t)\right\|=O\left(e^{t \omega}\right) \quad \text { as } t \longrightarrow \infty
$$

then $u(t)$ is called a solution to $\left(A C P_{\omega} ; A, x\right)\left(\left(A C P_{\omega} ; x\right), A C P_{\omega}\right)$. Let $Y$ be a linear subspace of $X$. Then we say that $(A C P ; A, T, Y)((A C P$; $T, Y), A C P)$ is well posed if for every $x \in Y$ there is a unique solution to $A C P$. Also, we say that $\left(A C P_{\omega} ; A, Y\right)\left(\left(A C P_{\omega} ; Y\right), A C P_{\omega}\right)$ is well posed if for every $x \in Y$ there is a unique solution to $A C P_{\omega}$.

In [12], Phillips gave a characterization of infinitesimal generators of $\left(C_{0}\right)$ semigroups in terms of $(A C P ; A, \infty, D(A))$ well posedness. The main purpose of this paper is to show that $\left(A C P ; A, T, D\left(A^{k+1}\right)\right)$ (or

Communicated by S. Matsuura, September 2, 1974.

* Department of Mathematics, School of Education, Okayama University, Okayama. 
$\left.\left(A C P_{\omega} ; A, D\left(A^{k+1}\right)\right)\right)$ well posedness leads to the conclusion that $A$ $\in G(k, T)$ (or $A \in G_{\omega}(k)$ ). Definitions of the classes $G(k, T)$ and $G_{\omega}(k)$ are given later. The classes $G(k, T)$ and $G_{\omega}(k)$ are introduced by Oharu [10] and it is proved in [10] that the class $G(0, \infty)\left(=\cup G_{\omega}(k)\right)$ is just the same as the class of infinitesimal generators of $\left(\stackrel{\omega}{C_{0}}\right)$ semigroups. The class $\underset{k, T}{\cup} G(k, T)$ (or $\cup_{\omega, k} G_{\omega}(k)$ ) is the same as the class of infinitesimal generators of regular distribution semigroups (or exponential distribution semigroups). See [10], [11], and Theorem 1.3 of this paper. In this sense, this paper may be considered as an extension of [12]. Correctly speaking, our results is as follows.

Main Theorem. Let $A$ be a densely defined closed linear operator with nonvacuous resolvent set $\rho(A)$, and let $T \in(0, \infty]$ and $k \in \mathbb{Z}^{+}$. If $\left(A C P ; A, T, D\left(A^{k+1}\right)\right)$ is well posed, then $A$ satisfies

(I) $\rho(A)$ contains a half line $\left\{\lambda \in \mathbf{R} ; \lambda \geqq \omega_{0}\right\}$ for some $\omega_{0} \in \mathbf{R}$,

(II) for every $\tau \in(0, T)$ there is a $C_{\tau}>0$ such that

$\sup \left\{\left\|\lambda^{n} R(\lambda ; A)^{n} x\right\| ; 0 \leqq n / \lambda \leqq \tau, \lambda \geqq \omega_{0}, n \in \mathbf{N}\right\} \leqq C_{\tau}\|x\|_{k}$

for every $x \in D\left(A^{k}\right)$.

Here $R(\lambda ; A)$ denotes the resolvent of $A$ at $\lambda \in \rho(A)$, and $\|x\|_{k}=\sum_{j=0}^{k}\left\|A^{j} x\right\|$, $A^{0} x=x$.

Theorem 1.2. Let $A$ be a densely defined closed linear operator with nonvacuous resolvent set $\rho(A)$, and let $\omega \in \mathbb{R}$ and $k \in \mathbb{Z}^{+}$. If $\left(A C P_{\omega} ; A, D\left(A^{k+1}\right)\right)$ is well posed, then $A$ satisfies

( $\left.\mathrm{I}_{\omega}\right) \rho(A)$ contains the half plane $\{\lambda \in C ; \operatorname{Re} \lambda>\omega\}$

$\left(\mathrm{II}_{\omega}\right)$ there is an $M>0$ such that

$$
\left\|R(\lambda ; A)^{n} x\right\| \leqq \frac{M}{(\operatorname{Re} \lambda-\omega)^{n}}\|x\|_{k}
$$

for every $x \in D\left(A^{k}\right), \lambda \in \mathbb{C}$ with $\operatorname{Re} \lambda>\omega$ and $n \in \mathbb{N}$.

Henceforth a linear operator $A$ will be called a regular operator 
if it is densely defined closed and the resolvent set is nonvoid. We shall denote by $G(k, T)$ (or $G_{\omega}(k)$ ) the set of all regular operators satisfying (I) and (II) (or $\left(\mathrm{I}_{\omega}\right)$ and $\left(\mathrm{II}_{\omega}\right)$ ). It is proved in [10] that, if $A$ $\in G(k, T)$ (or $\left.A \in G_{\omega}(k)\right)$, the exponential formula

$$
u(t ; x)=\lim _{n \rightarrow \infty}\left(1-\frac{t}{n} A\right)^{-n} x
$$

converges for $x \in D\left(A^{k}\right)$ and $t \in[0, T)$ (or $t \geqq 0$ ), and for $x \in D\left(A^{k+1}\right)$, $u(t ; x)$ of (E) gives the unique solution to $(A C P ; A, T, x)$ (or $\left(A C P_{\omega}\right.$; $A, x)$ ). Therefore, we have: Let $A$ be a regular operator. Then (ACP; $\left.A, T, D\left(A^{k+1}\right)\right)\left(\right.$ or $\left.\left(A C P_{\omega} ; A, D\left(A^{k+1}\right)\right)\right)$ is well posed if and only if $A \in G(k, T)$ (or $\left.A \in G_{\omega}(k)\right)$.

The relationship between classes $G(k, T)$ and $G_{\omega}(k)$, and the class of infinitesimal generators of distribution semigroups is studied in [10] and [11]: (1) $A$ is the infinitesimal generator of a regular distribution semigroup (R. D. S. G.) if and only if for every $T \in(0, \infty)$ there is a $k(T) \in \mathbb{Z}^{+}$such that $A \in G(k(T), T)$. (2) $A$ is the infinitesimal generator of an exponential distribution semigroup if and only if $A \in G_{\omega}(k)$ for some $\omega \in \mathbb{R}$ and $k \in \mathbb{Z}^{+}$.

Our second purpose is to reform "if" part of (1).

Theorem 1.3. If $A \in G(k, T)$ for some $k \in \mathbb{Z}^{+}$and $T \in(0, \infty]$, then $A$ is the infinitesimal generator of an R.D.S.G.

Detailed explanations concerning R. D.S. G. will be seen in [3], [4], [8], [10], [11], [16], and [17].

This paper consists of five sections. Section 2 contains fundamental lemmas which are based on the closed graph theorem. These lemmas are used throughout this paper. In Section 3, we prove Theorem 1.2. The proof is easy because of (1.1). We prove $\left(I_{\omega}\right)$ and then we show the relation $R(\lambda ; A) x=\int_{0}^{\infty} e^{-\lambda t} u(t ; x) d t . \quad\left(\mathrm{II}_{\omega}\right)$ follows from this relation. However, in the proof of Main Theorem, this relation may not be employed. Because it is not assumed that $A C P$ is well posed in the sense of semigroup (see [13; p. 396] and [7; Chap. 1]) or $A C P$ is Hadamard correct (see [9] and Theorem 1.2 of this paper), and so the growth order of the solution $u(t)$ of $A C P$ is not necessarily estimated 
as (1.1). Also, ACP considered in Main Theorem does not necessarily become well posed in the sense of semigroup (see [12] and [14]). Therefore, we must employ another approach. In Section 4, we show that the resolvent set of the regular operator $A$ contains a logarithmic region if $A C P$ is well posed. An estimate for the resolvent of $A$ is given under the same assumptions. See Proposition 4.1. As a consequence of Proposition 4.1 and [8], we obtain Theorem 1.3. Proposition 4.1 is also used in the proof of Main Theorem. Finally, in Section 5, we complete the proof of Main Theorem.

In the preparation of this paper, the author received many valuable suggestions and constant encouragement from Professor H. Sunouchi and Professor I. Miyadera. My thanks also go to Professor K. Kojima and Professor S. Oharu. Without their advice and interests in this work this paper could not be accomplished.

\section{Preliminaries}

In this paper, $\mathbf{R}$ denotes the real line, and $\mathbf{C}$ denotes the complex plane. $\operatorname{Re} \lambda$ and $\operatorname{Im} \lambda$ denote the real part and the imaginary part of $\lambda \in \mathbf{C}$ respectively. $\mathbf{N}=\{1,2, \ldots\}$ and $\mathbf{Z}^{+}=\mathbf{N} \cup\{0\}$. Let $I$ be an interval and let $Y$ be a Banach space. We denote by $C^{j}(I: Y)$ the set of all $Y$-valued $j$-times continuously differentiable functions defined on $I$. $C(I ; Y)$ denotes the set of all continuous functions. If there is no ambiguity, we write $C^{j}(Y)$. Let $A$ be a closed linear operator in a Banach space $X . \rho(A)$ and $R(\lambda ; A)$ denote the resolvent set and the resolvent of $A$ respectively. $D\left(A^{j}\right)$ is often considered as a Banach space with respect to the norm $\|x\|_{j}=\sum_{i=0}^{j}\left\|A^{i} x\right\| ; A^{0} x=x$. This Banach space will be denoted by $\left[D\left(A^{j}\right)\right]$. Let $T \in(0, \infty]$ and $\omega \in \mathbf{R}$. We shall consider $C([0, T) ;[D(A)])$ as a Fréchet space $Z$ endowed with the system of seminorms $\left\{\sup _{0 \leqq t \leqq \tau}\|u(t)\|_{1} ; \tau \in(0, T)\right\}$. Also, we denote by $Z_{\omega}$ the Banach space consists of all elements $u \in C([0, \infty) ;[D(A)])$ such that $\sup _{t \geqq 0} e^{-t \omega}\|u(t)\|_{1}<\infty$. The norm of $Z_{\omega}$ is by definition $\sup _{t \geqq 0} e^{-t \omega}\|u(t)\|_{1}$. Let $k \in \mathbb{Z}^{+}$. If $\left(A C P ; A, T, D\left(A^{k+1}\right)\right)$ is well posed, $\{U(t)\}$ denotes the set of linear operators which assign each $x \in D\left(A^{k+1}\right)$ the solution $u(t ; x)=U(t) x$ of $A C P$, If $\left(A C P_{\omega} ; A, D\left(A^{k+1}\right)\right)$ is well 
posed, $U(t) x$ is changed to mean the solution of $A C P_{\omega}$. We call $\{U(t)\}$ the solution operators for $A C P$ (or $A C P_{\omega}$ ).

Now, we note that, $\left(A C P_{\omega} ; A, D\left(A^{k+1}\right)\right)$ is well posed if and only if $\left(A C P_{\omega+\omega^{\prime}} ; A+\omega^{\prime}, D\left(\left(A+\omega^{\prime}\right)^{k+1}\right)\right)$ is well posed. This implies that one may consider as $\omega>0$ in Theorem 1.2. Therefore, we consider as $\omega>0$ whenever it is assumed that $A C P_{\omega}$ is well posed. This remark is used in the proof of Lemma 2.1 .

Lemma 2.1. Let $A$ be a closed linear operator. If $(A C P ; T$, $\left.D\left(A^{k+1}\right)\right)$ or $\left(A C P_{\omega} ; D\left(A^{k+1}\right)\right)$ is well posed, then for every $j \in \mathbf{N}$ and $x \in D\left(A^{k+j+1}\right)$, we have $U(t) x \in D\left(A^{j}\right), U(\cdot) x \in C^{j}(X)$ and

$$
\left(\frac{d}{d t}\right)^{j} U(t) x=A^{j} U(t) x=U(t) A^{j} x
$$

Proof. We shall prove the case $j=1$. The general case is proved by induction. For every $x \in D\left(A^{k+2}\right)$, we have $(d / d t) U(t) A x=A U(t) A x$. Integrating this equation on $[0, t]$, and using the closedness of $A$, we have

$$
U(t) A x=A x+\int_{0}^{t} A U(s) A x d s=A z(t),
$$

where $z(t)=x+\int_{0}^{t} U(s) A x d s \in D(A)$. It is easy to see that $(d / d t) z(t)=$ $U(t) A x=A z(t)$ and $z(0)=x$. Therefore, $z(t)$ is the solution to $A C P$. In the case of $A C P_{\omega}$, we have to show that the solution $z(t)$ satisfies (1.1). But, since $\omega>0$ and $\|U(s) A x\| \leqq M e^{s \omega}$ for some $M>0$, we have

$$
\begin{gathered}
\|z(t)\|+\left\|z^{\prime}(t)\right\| \leqq\|x\|+M\left(e^{t \omega}-1\right) / \omega \\
=O\left(e^{t \omega}\right), \quad \text { as } \quad t \longrightarrow \infty .
\end{gathered}
$$

Thus $z(t)$ is the solution to $A C P_{\omega}$. Therefore, in either case, we have $z(t)=U(t) x$, by the uniqueness of the solution, and by (2.1), we have $U(t) A x=A U(t) x$.

Lemma 2.2. Let $A$ be a closed linear operator with $\rho(A) \neq \phi$.

(a) If $\left(A C P ; T, D\left(A^{k+1}\right)\right)$ is well posed, then for every $\tau \in(0, T)$, there is an $M_{\tau}>0$ such that 


$$
\|U(t) x\| \leqq M_{\tau}\|x\|_{k}
$$

for every $t \in[0, \tau]$ and $x \in D\left(A^{k+1}\right)$.

(b) If $\left(A C P_{\omega} ; D\left(A^{k+1}\right)\right)$ is well posed, then there is an $M>0$ such that

$$
\|U(t) x\| \leqq M e^{t \omega}\|x\|_{k}
$$

for every $t \geqq 0$ and $x \in D\left(A^{k+1}\right)$.

Proof. We prove (a). It is easy to see that the linear operator $x \mapsto U(\cdot) x$, defined on $\left[D\left(A^{k+1}\right)\right]$ with range $Z$, is a closed operator. Therefore, $x \mapsto U(\cdot) x$ is continuous by the closed graph theorem. Let $\lambda_{0} \in \rho(A)$ and $x \in D\left(A^{k+1}\right)$, and put $y=R\left(\lambda_{0} ; A\right) x$. Then, by Lemma 2.1, we have

$$
\|U(t) x\|=\left\|\left(\lambda_{0}-A\right) U(t) y\right\| \leqq \text { const. }\|U(t) y\|_{1},
$$

where constant is independent of $t$ and $x$. Since $U(\cdot)$ is continuous, for every $\tau \in(0, T)$, we have

$$
\|U(t) y\|_{1} \leqq \text { const. }(\tau)\|y\|_{k+1} \leqq \text { const. }(\tau)\|x\|_{k},
$$

for $t \in[0, \tau]$. This shows (2.2).

We can prove (b) in a same way as (a), if we replace $Z$ with $Z_{\omega}$.

Lemma 2.3. Let $A$ be a regular operator. Then $D\left(A^{m}\right)$ is dense in $\left[D\left(A^{n}\right)\right]$ for every $m>n, m, n \in \mathbf{N}$.

Lemma 2.3 is proved in [10; Lemma 2.7].

Lemmas 2.2 and 2.3 show that, if $A$ is a regular operator, we can extend $\{U(t)\}$ uniquely to a family of operators defined on $D\left(A^{k}\right)$ with range $X$ satisfying (2.2) (or (2.3)) for all $x \in D\left(A^{k}\right)$ and $t \in[0, \tau]$ (or $t$ $\geqq 0$ ); this extended family will be denoted by the same symbol $\{U(t)\}$, also it will be called the solution operators for $A C P$ (or $A C P_{\omega}$ ). Moreover, we see that $U(t) x$ is strongly continuous in $t \in[0, T)($ or $t \geqq 0)$ for every $x \in D\left(A^{k}\right)$. 


\section{Proof of Theorem $\mathbf{1 . 2}$}

Throughout this section, let $A$ be a regular operator, and let $\omega \in \mathbf{R}$ and $k \in \mathbf{Z}^{+}$. Suppose that $\left(A C P_{\omega} ; A, D\left(A^{k+1}\right)\right)$ is well posed, and let $\{U(t)\}$ be the solution operators for $A C P_{\omega}$. We define

$$
R_{0}(\lambda) x=\int_{0}^{\infty} e^{-\lambda t} U(t) x d t
$$

for $\operatorname{Re} \lambda>\omega$ and $x \in D\left(A^{k}\right)$.

Lemma 3.1. $R_{0}(\lambda)$ has a unique extension to a bounded linear operator; this extended operator will be denoted by $R(\lambda)$.

Proof. Let $\lambda_{0} \in \rho(A)$ and $x \in D\left(A^{k+1}\right)$, and put

$$
V_{m}^{k}(t)=\left(\lambda_{0}-A\right)^{m} U(t) R\left(\lambda_{0} ; A\right)^{k} x \quad \text { for } \quad 0 \leqq m \leqq k .
$$

Then we have

$$
\begin{aligned}
R_{0}(\lambda) x= & \int_{0}^{\infty} e^{-\lambda t} V_{k}^{k}(t) d t \\
= & \lambda_{0} \int_{0}^{\infty} e^{-\lambda t} V_{k-1}^{k}(t) d t-\int_{0}^{\infty} e^{-\lambda t} \frac{d}{d t} V_{k-1}^{k}(t) d t \\
= & \lambda_{0} \int_{0}^{\infty} e^{-\lambda t} V_{k-1}^{k}(t) d t+R\left(\lambda_{0} ; A\right) x \\
& +\int_{0}^{\infty} \frac{d}{d t}\left(e^{-\lambda t}\right) V_{k-1}^{k}(t) d t \\
= & \quad \ldots \ldots \ldots \ldots \ldots . . . . . . . . . \\
= & \int_{0}^{\infty}\left\{\sum_{i=0}^{k}\left(\begin{array}{c}
k \\
i
\end{array}\right) \lambda_{0}^{k-i}(-\lambda)^{i} e^{-\lambda t}\right\} V_{0}^{k}(t) d t \\
& +\sum_{i=0}^{k-1}\left\{\sum_{j=0}^{i}\left(\begin{array}{c}
i \\
j
\end{array}\right) \lambda_{0}^{i-j}(-\lambda)^{j}\right\} R\left(\lambda_{0} ; A\right)^{i+1} x \\
= & \left(\lambda_{0}-\lambda\right)^{k} \int_{0}^{\infty} e^{-\lambda t} V_{0}^{k}(t) d t
\end{aligned}
$$




$$
+\sum_{i=0}^{k-1}\left(\lambda_{0}-\lambda\right)^{i} R\left(\lambda_{0} ; A\right)^{i+1} x
$$

where $\left(\begin{array}{c}i \\ j\end{array}\right)$ denotes the binomial coefficient. Therefore, by Lemma 2.2, we have

$$
\left\|R_{0}(\lambda) x\right\| \leqq \frac{C}{\operatorname{Re} \lambda-\omega}(1+|\lambda|)^{k}\|x\|
$$

This completes the proof of Lemma 3.1 .

Proof of Theorem 1.2. For $x \in D\left(A^{k+1}\right)$ and $\operatorname{Re} \lambda>\omega$, we have $(\lambda$ $-A) R_{0}(\lambda) x=R_{0}(\lambda)(\lambda-A) x=x$. Therefore, by Lemmas 2.3 and 3.1, $(\lambda$ $-A) R(\lambda) x=x$ holds for every $x \in X$ and $R(\lambda)(\lambda-A) x=x$ holds for every $x \in D(A)$. This implies $\left(I_{\omega}\right)$. Furthermore, we have

$$
R(\lambda ; A) x=\int_{0}^{\infty} e^{-\lambda t} U(t) x d t
$$

for $\operatorname{Re} \lambda>\omega$ and $x \in D\left(A^{k}\right)$. Therefore, by Lemma 2.2, we have

$$
\begin{aligned}
\left\|R(\lambda ; A)^{n} x\right\| & =\left\|\frac{(-1)^{n-1}}{(n-1) !}\left(\frac{d}{d \lambda}\right)^{n-1} R(\lambda ; A) x\right\| \\
& \leqq \frac{1}{(n-1) !} \int_{0}^{\infty} e^{-t \operatorname{Re} \lambda} t^{n-1}\|U(t) x\| d t \\
& =\frac{M}{(\operatorname{Re} \lambda-\omega)^{n}}\|x\|_{k} .
\end{aligned}
$$

This completes the proof of Theorem 1.2.

\section{The Resolvent Set and the Resolvent of $A$}

Throughout this section, let $A$ be a regular operator and let $T \in(0$, $\infty]$ and $k \in \mathbf{Z}^{+}$. The main purpose of this section is to prove the following

Proposition 4.1. If $\left(A C P ; A, T, D\left(A^{k+1}\right)\right)$ is well posed, then the resolvent set of $A$ contains a region $\Omega$ of the form 


$$
\Omega=\left\{\lambda \in \mathbf{C} ; \operatorname{Re} \lambda \geqq \alpha_{1} \log |\operatorname{Im} \lambda|+\beta_{1}, \operatorname{Re} \lambda \geqq \omega_{1}\right\} \cup\left\{\lambda \in \mathbf{R} ; \lambda \geqq \omega_{1}\right\},
$$

where $\alpha_{1}, \beta_{1}$, and $\omega_{1}$ are positive numbers, and for $\lambda \in \Omega$, the resolvent of $A$ satisfies

$$
\|R(\lambda ; A)\| \leqq C(1+|\lambda|)^{k} .
$$

As a consequence of Proposition 4.1 and [8] ,we obtain

Corollary 4.2. Suppose that $\left(A C P ; A, T, D\left(A^{k+1}\right)\right)$ is well posed, then $A$ is the infinitesimal generator of an R. D.S. G.

Using Corollary 4.2, we obtain Theorem 1.3.

Proof of Theorem 1.3. Suppose that $A \in G(k, T)$. Then, by [10; Th. 2.8], $\left(A C P ; T, D\left(A^{k+1}\right)\right)$ is well posed. Therefore, Theorem 1.3 follows from Corollary 4.2 .

To prove Proposition 4.1, we need two lemmas; Lemmas 4.3 and 4.4. Now, we denote by $D(I)$ the usual Schwartz space on the open interval $I$. Let $\varphi \in D(-\infty, T)$ be a function satisfying $\varphi(t)=1$ on $[0$, $\delta]$ and $\varphi(t)=0$ on $[2 \delta, T)$, where $2 \delta \in(0, T)$, and put $\varphi_{\lambda}=e^{-\lambda t} \varphi$. Suppose that $\left(A C P ; T, D\left(A^{k+1}\right)\right)$ is well posed, and let $\{U(t)\}$ be the solution operators for $A C P$. We define

$$
R_{0}(\lambda) x=\int_{0}^{T} \varphi_{\lambda}(t) U(t) x d t
$$

for $x \in D\left(A^{k}\right)$ and $\operatorname{Re} \lambda \geqq 0$.

Lemma 4.3. $R_{0}(\lambda)$ has a unique extension to a bounded linear operator $R(\lambda)$. Moreover, we have

$$
\|R(\lambda)\| \leqq C(1+|\lambda|)^{k}
$$

for some $C>0$.

Proof. Let $\lambda_{0} \in \rho(A)$ and $x \in D\left(A^{k+1}\right)$, and put $V_{k}^{m}(t)=\left(\lambda_{0}-A\right)^{m}$. $\cdot U(t) R\left(\lambda_{0} ; A\right)^{k} x$ for $0 \leqq m \leqq k$. Then we have 


$$
\begin{aligned}
R_{0}(\lambda) x= & \int_{0}^{T} \varphi_{\lambda}(t) V_{k}^{k}(t) d t \\
= & \int_{0}^{T}\left\{\sum_{i=0}^{k}\left(\begin{array}{c}
k \\
i
\end{array}\right) \lambda_{0}^{k-i} \varphi_{\lambda}^{(i)}(t)\right\} V_{0}^{k}(t) d t \\
& +\sum_{i=0}^{k-1}\left\{\sum_{j=0}^{i}\left(\begin{array}{c}
i \\
j
\end{array}\right) \lambda_{0}^{i-j} \varphi_{\lambda}^{(j)}(0)\right\} R\left(\lambda_{0} ; A\right)^{i+1} x,
\end{aligned}
$$

where $\varphi_{\lambda}^{(i)}$ is the $i$-th derivative of $\varphi_{\lambda}$. Since $\left\|U(t) R\left(\lambda_{0} ; A\right)^{k} x\right\| \leqq C\|x\|$ for $t \in[0,2 \delta]$, we have

$$
\begin{aligned}
\left\|R_{0}(\lambda) x\right\| \leqq & \text { const. }(1+|\lambda|)^{k} \int_{0}^{2 \delta}\left\|U(t) R\left(\lambda_{0} ; A\right)^{k} x\right\| d t \\
& + \text { const. }(1+|\lambda|)^{k-1}\|x\| \\
\leqq & \text { const. }(1+|\lambda|)^{k}\|x\| .
\end{aligned}
$$

This completes the proof of Lemma 4.3.

Let $x \in D\left(A^{k+1}\right)$. Then we have $(\lambda-A) R_{0}(\lambda) x=R_{0}(\lambda)(\lambda-A) x=(1$ $\left.+Q_{0}(\lambda)\right) x$, where $Q_{0}(\lambda) x=\int_{0}^{T} e^{-\lambda t} \varphi^{\prime}(t) U(t) x d t$

Lemma 4.4. $Q_{0}(\lambda)$ has a unique extension to a bounded linear operator $Q(\lambda)$. Moreover we have

$$
\|Q(\lambda)\| \leqq C(1+|\lambda|)^{k} e^{-\delta \operatorname{Re} \lambda}
$$

for some $C>0$, and for every $\lambda$ in a half plane of the form $\{\lambda \in \mathbb{C}$; $\left.\operatorname{Re} \lambda \geqq \omega_{1}>0\right\}$.

Proof. Let $\lambda_{0} \in \rho(A)$ and $x \in D\left(A^{k+1}\right)$. Then, since $\varphi^{\prime}(t) \in D(\delta, T)$, we have

$$
\begin{aligned}
Q_{0}(\lambda) x & =\int_{0}^{T} e^{-\lambda t} \varphi^{\prime}(t)\left(\lambda_{0}-A\right)^{k} U(t) R\left(\lambda_{0} ; A\right)^{k} x d t \\
& =\sum_{j=0}^{k}\left(\begin{array}{c}
k \\
j
\end{array}\right) \lambda_{0}^{k-j} \int_{0}^{T} e^{-\lambda t} \varphi^{\prime}(t)\left(-\frac{d}{d t}\right)^{j} U(t) R\left(\lambda_{0} ; A\right)^{k} x d t \\
& =\sum_{j=0}^{k}\left(\begin{array}{c}
k \\
j
\end{array}\right) \lambda_{0}^{k-j}\left\{\sum_{i=0}^{j}\left(\begin{array}{l}
j \\
i
\end{array}\right)(-\lambda)^{j-i} \int_{0}^{T} e^{-\lambda t} \varphi^{(i+1)}(t) U(t) R\left(\lambda_{0} ; A\right)^{k} x d t\right\} .
\end{aligned}
$$


This implies that $Q_{0}(\lambda)$ can be uniquely extended to a bounded linear operator $Q(\lambda)$, and since $\varphi^{(i+1)} \in D(\delta, 2 \delta)$, we have

$$
\begin{aligned}
\|Q(\lambda) x\| & \leqq \text { const. }(1+|\lambda|)^{k} \sum_{i=0}^{k} \int_{\delta}^{2 \delta} e^{-t \operatorname{Re} \lambda}\left|\varphi^{(i+1)}(t)\right|\left\|U(t) R\left(\lambda_{0} ; A\right)^{k} x\right\| d t \\
& \leqq \text { const. }(1+|\lambda|)^{k}\left(\int_{\delta}^{\infty} e^{-t \operatorname{Re} \lambda} d t\right)\|x\| \\
& \leqq \text { const. }(1+|\lambda|)^{k} e^{-\delta \operatorname{Re} \lambda\|x\|}
\end{aligned}
$$

for every $\lambda$ with $\operatorname{Re} \lambda \geqq \omega_{1}>0$, and for every $x \in X$.

Proof of Proposition 4.1. By Lemmas 4.3 and 4.4, we have

$$
\begin{cases}(\lambda-A) R(\lambda) x=(1+Q(\lambda)) x & \text { for } \quad x \in X, \\ R(\lambda)(\lambda-A) x=(1+Q(\lambda)) x & \text { for } \quad x \in D(A) .\end{cases}
$$

If $\|Q(\lambda)\| \leqq 1 / 2$, then $1+Q(\lambda)$ is invertible and $(1+Q(\lambda))^{-1}$ is a bounded linear operator satisfying $\left\|(1+Q(\lambda))^{-1}\right\| \leqq 2$. Therefore, Lemmas 4.3 and 4.4, and (4.3) implies that

$$
\begin{aligned}
\Omega_{0} & =\left\{\lambda \in \mathbf{C} ; C(1+|\lambda|)^{k} e^{-\delta \operatorname{Re} \lambda} \leqq 1 / 2, \operatorname{Re} \lambda \geqq \omega_{1}\right\} \\
& \subset\left\{\lambda \in \mathbf{C} ;\|Q(\lambda)\| \leqq 1 / 2, \operatorname{Re} \lambda \geqq \omega_{1}\right\} \\
& \subset \rho(A),
\end{aligned}
$$

and for $\lambda \in \Omega_{0}$, we have

$$
\begin{aligned}
\|R(\lambda ; A)\| & =\left\|(1+Q(\lambda))^{-1} R(\lambda)\right\| \\
& \leqq \text { const. }(1+|\lambda|)^{k}
\end{aligned}
$$

On the other hand, region $\Omega_{0}$ contains a region $\Omega$ of the form (4.1). This completes the proof of Proposition 4.1.

\section{Proof of Main Theorem}

Throughout this section, we assume the hypotheses of Main Theorem. Note that, by Proposition 4.1, the resolvent set of $A$ contains a region 
$\Omega$ of the form (4.1), and for $\lambda \in \Omega$, the resolvent of $A$ satisfies (4.2). Therefore, condition (I) is already shown.

Now, let $\{U(t)\}$ be the solution operators for $A C P$. For any $\tau_{0} \in(0, T)$ and $x \in D\left(A^{k+1}\right)$, we have

$$
\begin{aligned}
A \int_{0}^{\tau_{0}} e^{-\lambda t} U(t) x d t & =\int_{0}^{\tau_{0}} e^{-\lambda t} \frac{d}{d t} U(t) x d t \\
& =e^{-\lambda \tau_{0}} U\left(\tau_{0}\right) x-x+\lambda \int_{0}^{\tau_{0}} e^{-\lambda t} U(t) x d t
\end{aligned}
$$

If $\lambda \in \Omega$, we have

$$
R(\lambda ; A) x=\int_{0}^{\tau_{0}} e^{-\lambda t} U(t) x d t+e^{-\lambda \tau_{0}} R(\lambda ; A) U\left(\tau_{0}\right) x
$$

for every $x \in D\left(A^{k}\right)$. Let $\tau$ and $\varepsilon$ be any positive numbers such $\tau_{0}>(1$ $+\varepsilon) \tau$. Take, $\alpha>\max \left\{\alpha_{1},(k+2) /\left(\tau_{0}-(1+\varepsilon) \tau\right)\right\}, \beta>\beta_{1}, \omega_{2}>\max \left(\omega_{1}, \beta\right\}$ and $\eta_{0}=\exp \left(\left(\omega_{2}-\beta\right) / \alpha\right)(>1)$, and let $\Gamma=\Gamma_{1} \cup \Gamma_{2}$ be a contour of the form

$$
\begin{aligned}
& \Gamma_{1}=\left\{\zeta=\xi+i \eta ; \xi=\alpha \log |\eta|+\beta,|\eta| \geqq \eta_{0}\right\}, \\
& \Gamma_{2}=\left\{\zeta=\omega_{2}+i \eta ;|\eta| \leqq \eta_{0}\right\},
\end{aligned}
$$

oriented from the lower to upper half plane. Here, $\alpha_{1}, \beta_{1}$, and $\omega_{1}$ are positive numbers mentioned in (4.1), and $i$ denotes the imaginary unit. Note that $\Gamma \subset \Omega$. By Cauchy's integral formula, we have

$$
e^{-\lambda \tau_{0}} R(\lambda ; A) U\left(\tau_{0}\right) x=\frac{1}{2 \pi i} \int_{\Gamma} \frac{1}{\lambda-\zeta} e^{-\zeta \tau_{0}} R(\zeta ; A) U\left(\tau_{0}\right) x d \zeta
$$

for $\lambda>\omega_{2}$. Hence, we have

$$
\begin{aligned}
\lambda^{n} R(\lambda ; A)^{n} x= & \frac{(-1)^{n-1} \lambda^{n}}{(n-1) !}\left(\frac{d}{d \lambda}\right)^{n-1} R(\lambda: A) x \\
= & \frac{\lambda^{n}}{(n-1) !} \int_{0}^{\tau_{0}} e^{-\lambda t} t^{n-1} U(x) x d t \\
& +\frac{1}{2 \pi i} \int_{\Gamma} e^{-\zeta \tau_{0}}\left(1-\frac{\zeta}{\lambda}\right)^{-n} R(\zeta ; A) U\left(\tau_{0}\right) x d \zeta \\
\equiv & I_{1}+I_{2}
\end{aligned}
$$


for every $n \in \mathbf{N}, \lambda>\omega_{2}$ and $x \in D\left(A^{k}\right)$.

Lemma 5.1. There is an $M>0$ such that

$$
\left\|I_{1}\right\| \leqq M\|x\|_{k}
$$

for all $x \in D\left(A^{k}\right), n \in \mathbf{N}$ and $\lambda>0$.

Proof. By Lemma 2.2, there is an $M=M_{\tau_{0}}>0$ such that $\|U(t) x\|$ $\leqq M\|x\|_{k}$. Therefore, we have

$$
\begin{aligned}
\left\|I_{1}\right\| & \leqq \frac{\lambda^{n}}{(n-1) !}\left(\int_{0}^{\infty} e^{-\lambda t} t^{n-1} d t\right) M\|x\|_{k} \\
& =M\|x\|_{k} .
\end{aligned}
$$

Lemma 5.2. For every $\varepsilon \in(0,1)$, there is a $C_{\varepsilon}>0$ such that

$$
\left|\left(1-\frac{\zeta}{\lambda}\right)\right|^{-n} \leqq C_{\varepsilon} e^{(1+\varepsilon) \tau \xi}
$$

for $\zeta=\xi+i \eta \in \Gamma, n / \lambda \in[0, \tau], \lambda \geqq \omega_{0}>2 \omega_{2}$ and $n \in \mathbf{N}$.

Proof. For every $\varepsilon \in(0,1)$, there is a $\delta=\delta_{\varepsilon} \in(0,1 / 2]$ such that $(1-t)^{-1} \leqq e^{(1+\varepsilon) t}$ for $t \in[0, \delta]$. Therefore, we have

$$
\left\{\begin{array}{l}
\left|\left(1-\frac{\zeta}{\lambda}\right)^{-n}\right| \leqq\left|1-\frac{\xi}{\lambda}\right|^{-n} \leqq e^{n(1+\varepsilon) \xi / \lambda} \leqq e^{(1+\varepsilon) \xi \tau} \\
\text { for } \xi / \lambda \in[0, \delta] \text { and } n / \lambda \in[0, \tau]
\end{array}\right.
$$

In the case when $\xi \mid \lambda>\delta$, we have

$$
\begin{aligned}
\left|\left(1-\frac{\zeta}{\lambda}\right)^{-1}\right| & =\left|\frac{\lambda}{\lambda-\zeta}\right| \leqq \frac{\lambda}{|\eta|} \leqq \frac{\xi}{\delta|\eta|} \\
& \leqq \frac{\alpha \log |\eta|+\beta}{\delta|\eta|}
\end{aligned}
$$

for $\zeta=\xi+i \eta \in \Gamma_{1}$. Therefore, if $\left|(1-(\zeta / \lambda))^{-1}\right|>1, \zeta=\xi+i \eta \in \Gamma_{1}, n / \lambda \in[0$, $\tau]$ and $n \in \mathbf{N}$, we have 


$$
\begin{aligned}
\left|\left(1-\frac{\zeta}{\lambda}\right)^{-n}\right| & \leqq\left(\frac{\alpha \log |\eta|+\beta}{\delta|\eta|}\right)^{n} \\
& \leqq\left(\frac{\alpha \log |\eta|+\beta}{\delta|\eta|}\right)^{\tau \lambda} \leqq\left(\frac{\alpha \log |\eta|+\beta}{\delta|\eta|}\right)^{\xi \tau / \delta} \\
& \leqq\left(\frac{\alpha \log |\eta|+\beta}{\delta|\eta|}\right)^{\tau(\alpha \log |\eta|+\beta) / \delta}
\end{aligned}
$$

Hence, we have

$$
\left.\sup \left\{\left|\left(1-\frac{\zeta}{\lambda}\right)^{-n}\right| ; \zeta=\xi+i \eta \in \Gamma_{1}, n|\lambda \in[0, \tau], \xi| \lambda>\delta\right\}\right\}<\infty
$$

On the other hand, we have

$$
\left\{\begin{array}{l}
\left|\left(1-\frac{\zeta}{\lambda}\right)^{-n}\right| \leqq\left|1-\frac{\omega_{2}}{\lambda}\right|^{-n} \leqq e^{2 \omega_{2} n / \lambda} \leqq e^{2 \omega_{2} \tau} \\
\text { for } \zeta=\omega_{2}+i \eta \in \Gamma_{2}, \lambda \geqq \omega_{0}\left(>2 \omega_{2}\right) \\
\text { and } n / \lambda \in[0, \tau] .
\end{array}\right.
$$

Combining (5.1), (5.2) and (5.3), we get Lemma 5.2.

Proof of Main Theorem. By Lemma 5.2, we have

$$
\left\|I_{2}\right\| \leqq C\left(\int_{\Gamma} e^{-\xi \tau_{0}+(1+\varepsilon) \xi \tau}\left\|R(\zeta ; A) U\left(\tau_{0}\right) x\right\||d \zeta|\right)
$$

In view of Lemma 2.2, (4.2) and the definition of $\Gamma$, we have

$$
\begin{aligned}
\left\|I_{2}\right\| \leqq & C\left(\int_{\Gamma} e^{-\left(\tau_{0}-(1+\varepsilon) \tau\right) \xi}(1+|\zeta|)^{k}|d \zeta|\right)\|x\|_{k} \\
\leqq & C\|x\|_{k}\left\{\int_{\eta_{0}}^{\infty} e^{-\left(\tau_{0}-(1+\varepsilon) \tau\right)(\alpha \log \eta+\beta)}(1+\eta)^{k} \eta^{-1} d \eta\right. \\
& \left.+\int_{-\eta_{0}}^{\eta_{0}} e^{-\omega_{2}\left(\tau_{0}-(1+\varepsilon) \tau\right)}(1+|\eta|)^{k} d \eta\right\} \\
\leqq & C x \|_{k} .
\end{aligned}
$$

Combining this estimate for $I_{2}$ and Lemma 5.1, we have 


$$
\sup \left\{\left\|\lambda^{n} R(\lambda ; A)^{n} x\right\| ; 0 \leqq n / \lambda \leqq \tau, \lambda \geqq \omega_{0}, n \in N\right\} \leqq C\|x\|_{k}
$$

for some $C>0$ which is independent of $x \in D\left(A^{k}\right)$. Since $\tau$ is an arbitrary number in $(0, T)$, we have (II). This completes the proof of Main Theorem.

\section{References}

[1] Beals, R., On the abstract Cauchy problem, J. Functional Analysis, 10 (1972), 281-299.

[2] - Semigroups and abstract Gevery spaces, J. Functional Analysis, 10 (1972), 300-308.

[3] Chazarain, J., Problemes du Cauchy abstraits et applications a quelques problemes mixtes, J. Functional Analysis, 7 (1971), 386-446.

[4] Fujiwara, D., A characterization of exponential distribution semigroups, $J$. Math. Soc. Japan, 18 (1966), 267-274.

[5] Hille E. and Phillips, R.S., Functional Analysis and Semigroups, Amer. Math. Soc. Colloq. Publ., 31 (1957).

[6] Komura, T., Semigroups of operators in a locally convex space, J. Functional Analysis, 2 (1968), 258-296.

[7] Krein, S. G., Linear differential equations in Banach spaces (in Russian), Nauka, 1967.

[8] Lions, J., Les semi-groups distributions, Portugal. Math., 19 (1960), 141-164.

[9] Miyadera, I., Oharu, S. and Okazawa, N., Generation theorems of seni-groups of linear operators, Publ. RIMS, Kyoto Univ., 8 (3) (1972), 509-555.

[10] Oharu, S., Semigroups of linear operators in a Banach space, Publ. RIMS, Kyoto Univ., 7 (2) (1971), 205-260.

[11] — Eine Bemerkung zur Charakterisierung der Distributionenhalbgruppen, Math. Ann., 204 (1973), 189-198.

[12] Phillips, R. S., A note on the abstract Cauchy problem, Proc. Nat. Acad. Sci. U.S.A., 40 (1954), 244-248.

[13] Sunouchi, H., Convergence of semi-discrete difference schemes of Cauchy problems, Tôhoku Math. J., 22 (1970), 394-408.

[14] Sanekata, N., A note on the abstract Cauchy problem in a Banach space, Proc. Japan Acad., 49 (1973), 510-513.

[15] - On the abstract Cauchy problem, Kôkyûroku, RIMS, Kyoto Univ., 184 (1973), 63-75 (in Japanese).

[16] Ushijima, T., Some properties of regular distribution semigroups, Proc. Japan Acad., 45 (1969), 224-227.

[17] - On the generation and smoothness of semigroups of linear operators, J. Fac. Sci., Univ. Tokyo, Sect. IA, 19 (1) (1972), 65-127.

[18] Yosida, K., Functional Analysis, Springer, Berlin, 1965. 
